\title{
Descarte de bolsas de sangue e prevalência de doenças infecciosas em doadores de sangue da Fundação Pró-Sangue/Hemocentro de São Paulo
}

\author{
Nanci A. Salles, ${ }^{1}$ Ester C. Sabino, ${ }^{1}$ Claudia C. Barreto, ${ }^{1}$ \\ Angela M. E. Barreto, ${ }^{1}$ Marcia M. Otani ${ }^{1}$ e Dalton F. Chamone ${ }^{1,2}$
}

RESUMO Objetivo. Analisar a evolução, de 1991 a 2001, do descarte sorológico na Fundação PróSangue/Hemocentro de São Paulo, o maior banco de sangue da América Latina, e verificar a prevalência de doenças infecciosas entre doadores dessa instituição no ano de 2001.

Métodos. Foram compilados os dados de descarte sorológico relativos aos anos de 1991 a 2001. Para determinar a prevalência de doenças infecciosas, foram analisadas 9942 amostras triadas em novembro de 2001, sendo as amostras reativas submetidas a testes confirmatórios. Resultados. Foi encontrada uma diminuição percentual significativa de descarte, de $20 \%$ em 1991 para 9\% em 2001. A prevalência de doenças infecciosas entre doadores em 2001 foi de $0,04 \%$ para vírus da imunodeficiência humana (VIH); 0,21\% para vírus da hepatite C (VHC); $0,06 \%$ para vírus T-linfotrópico humano (HTLV); para vírus da hepatite B (VHB), as prevalências foram de $0,14 \%$ para anti-HBc $+H B s A g, 1,68 \%$ para anti-HBc + anti-HBs e $1,67 \%$ para anti-HBc isolado; $1,10 \%$ para sifilis; e $0,14 \%$ para doença de Chagas.

Conclusão. A diminuição no descarte e a prevalência de doenças infecciosas entre doadores da Fundação Pró-Sangue/Hemocentro de São Paulo em 2001 refletem o aumento na porcentagem de doadores de repetição nesse banco de sangue.

Palavras-chave Bancos de sangue, doadores de sangue, testes sorológicos.

A Fundação Pró-Sangue/Hemocentro de São Paulo, o maior banco de sangue da América Latina, coleta, em média, 200000 bolsas de sangue ao ano, sendo responsável pelo fornecimento de $50 \%$ do sangue utilizado pela população

\footnotetext{
Fundação Pró-Sangue/Hemocentro de São Paulo, São Paulo (SP), Brasil. Correspondência e pedidos de separatas devem ser enviados a Ester C. Sabino no seguinte endereço: Fundação Pró-Sangue/ Hemocentro de São Paulo, Avenida Dr. Enéas de Carvalho Aguiar 155, $1^{\circ}$ andar, CEP 05409-010, São Paulo, SP, Brasil. Fax: +55-11-3088-8317; e-mail: sabinoec@uol.com.br

2 Universidade de São Paulo, Faculdade de Medicina, Departamento de Hematologia e Hemoterapia, São Paulo (SP), Brasil.
}

da Cidade de São Paulo (em torno de 18000000 de habitantes em 2002). Com o desenvolvimento acentuado da biotecnologia nos últimos anos, os testes sorológicos utilizados em bancos de sangue foram redesenhados para apresentar alta sensibilidade. Conseqüentemente, esses testes passaram a garantir uma melhor qualidade ao sangue transfundido. Houve também melhora em relação à especificidade desses testes (1-3).

A taxa de descarte sorológico nos bancos de sangue no Brasil varia de 10 a $20 \%$ (4). Esse índice é mais alto do que nos países desenvolvidos (5), prin- cipalmente devido à alta porcentagem de pessoas que doam sangue pela primeira vez e que apresentam uma prevalência de infecção próxima à da população em geral. Outro fator que contribui para essa problemática é a freqüente troca na marca dos testes de triagem sorológica utilizados, em função das normas de licitação a que estão subordinados os bancos de sangue públicos (como é o caso da Fundação Pró-Sangue) para a compra de tais produtos. Esse fenômeno ocorre mesmo quando são usados testes de alta sensibilidade e especificidade. Cada marca de teste gera resultados 
falso-positivos em amostras diferentes. Com isso, quando a marca dos reagentes é freqüentemente trocada, ocorre uma somatória dos resultados falso-positivos - em outras palavras, aumenta a chance de um doador sadio ter reatividade cruzada a pelo menos uma das marcas utilizadas. Esse fenômeno não aconteceria se a mesma marca fosse sempre utilizada (6).

Atualmente, existem poucos estudos na literatura mundial sobre a prevalência de doenças transmissíveis pelo sangue na população brasileira de doadores. Isso se deve ao fato de que a realização de testes confirmatórios em amostras reativas pelos métodos de triagem sorológica não é obrigatória em nosso país. A maioria dos estudos enfoca apenas alguns marcadores específicos (7-11). Assim, o objetivo deste estudo foi identificar a evolução do descarte sorológico na Fundação Pró-Sangue de 1991 a 2001 e verificar a prevalência de doenças infecciosas entre doadores dessa instituição no ano de 2001.

\section{MATERIAIS E MÉTODOS}

Para determinar as taxas de descarte sorológico da Fundação Pró-Sangue/ Hemocentro de São Paulo, analisamos todos os dados disponíveis para o período de $1^{\circ}$ de janeiro de 1991 a 31 de dezembro de 2001. A prevalência, por sua vez, foi analisada a partir da coleta de 9942 amostras no período de $1^{\circ}$ a 15 de novembro de 2001. Todas as 9942 amostras foram submetidas aos seguintes testes de triagem, conforme a rotina do banco de sangue: um teste imunoenzimático (enzyme immunoassay, EIA) para vírus T-linfotrópico humano (HTLV), vírus da hepatite $C$ (VHC) e da hepatite B (HBsAg e anti$\mathrm{HBc}$ ); dois testes EIA simultâneos para vírus da imunodeficiência humana (VIH); dois testes simultâneos para sífilis (EIA e Venereal Disease Research Laboratory slide test, VDRL); e três testes simultâneos para a doença de Chagas: EIA, imunofluorescência indireta (IFI) e hemaglutinação indireta (HAI). As amostras inicialmente reativas a um teste foram testadas novamente em duplicata para aquele parâmetro. Se a reatividade fosse mantida em pelo menos uma das replicatas, a bolsa era descartada e a amostra enviada para confirmação. Os testes utilizados, assim como o algoritmo de confirmação, são descritos a seguir para cada doença.

\section{VIH}

As amostras foram triadas com o uso simultâneo dos testes Vironostic $\mathrm{VIH}^{\circledR}$ Uni-Form plus O (OrganonTeknika, Boxtel, Holanda) e ORTHO ${ }^{\circledR}$ VIH-1/VIH-2 Ab-Capture ELISA Test (Ortho-Clinical Diagnostic, Raritan, EUA). As amostras reativas foram submetidas ao teste confirmatório de Western blot utilizando o ensaio VIH BLOT 2.2 (Genelabs ${ }^{\circledR}$ Diagnostics, Cingapura). Foram consideradas positivas se apresentassem ao menos uma banda do gene envelope (ENV) (gp41 ou gp120/160) além da banda p24 (GAG).

\section{VHC}

A presença de anticorpos anti-VHC foi analisada pelo teste Murex antiVHC (versão 4.0) (Murex Biotech S.A., Kyalami, África do Sul). A confirmação diagnóstica foi feita com immunoblot CHIRON* RIBA* VHC 3.0 S/A (Chiron Corporation, Emeryville, EUA), que exige a presença de, pelo menos, duas bandas com intensidade de uma cruz ou mais.

\section{HTLV tipo I e II}

As amostras foram triadas utilizando o teste ORTHO ${ }^{\circledR}$ HTLV-I/ HTLV-II AbCapture ELISA Test Systems (OrthoClinical Diagnostic, Raritan, EUA). Os exames reativos foram confirmados pelo método de Western blot HTLV BLOT 2.4 (Genelabs ${ }^{\circledR}$ Diagnostics, Cingapura), sendo consideradas positivas as amostras que apresentassem a banda GD21 e pelo menos uma das bandas do gene GAG (p19 ou p24).

\section{VHB}

A triagem sorológica do vírus da hepatite $B$ foi realizada através do uso simultâneo dos ensaios Hepanostika ${ }^{\circledR}$ HBsAg Uni-Form II e Hepanostika ${ }^{\circledR}$ anti-HBc Uni-Form (Organon-Teknika, Boxtel, Holanda). As amostras reativas ao anti-HBc e não reativas ao $\mathrm{HBsAg}$ foram testadas para verificar a presença de anticorpos anti-HBs (Radim, Roma, Itália).

\section{Sífilis}

A presença de anticorpos antiTreponema pallidum nestas amostras foi analisada pelo uso simultâneo do teste de VDRL (Omega Diagnostics Limited, Alloa, Escócia) e pelo EIA TREPANOSTIKA ${ }^{\circledR}$ TP (OrganonTeknika, Boxtel, Holanda). As amostras reativas a qualquer um desses testes foram submetidas ao teste confirmatório de imunofluorescência indireta (FTA-Abs) Fluoline $\mathrm{H}^{\circledR}$ (Biolab Diagnóstica S.A., Rio de Janeiro, Brasil).

\section{Doença de Chagas}

A triagem para doença de Chagas foi realizada pelo uso simultâneo dos testes HAI Hemacruzi (Biolab-Merieux S. A., Rio de Janeiro, Brasil), para soro em diluição de 1/20; IFI IMUNOCRUZI ${ }^{\circledR}$ (Biolab-Merieux Diagnóstica S.A., Rio de Janeiro, Brasil), para soro em diluição de 1/40; e EIA Hemobio Chagas (Embrabio S.A., São Paulo, Brasil). Foram consideradas positivas as amostras reativas aos três testes.

\section{RESULTADOS}

A tabela 1 descreve a taxa de descarte sorológico anual para cada doença no período de 1991 a 2001 na Fundação Pró-Sangue/Hemocentro de São Paulo. A partir desses dados, foi possível verificar uma diminuição percentual significativa de descarte nesse período. Ficou também evidente o súbito aumento de bolsas descarta- 
TABELA 1. Taxa de descarte sorológico anual para VIH, VHC, HTLV, VHB, sífilis e a doença de Chagas na Fundação Pró-Sangue/Hemocentro de São Paulo, Brasil, 1991 a 2001

\begin{tabular}{|c|c|c|c|c|c|c|c|c|c|c|c|}
\hline Teste & 1991 & 1992 & 1993 & 1994 & 1995 & 1996 & 1997 & 1998 & 1999 & 2000 & 2001 \\
\hline \multicolumn{12}{|l|}{ VIH } \\
\hline Vironostic & 0,1 & 0,3 & 0,2 & 0,4 & 0,4 & 0,7 & 0,4 & 0,2 & 0,2 & 0,1 & 0,1 \\
\hline Vironostic + ELISA & 0,5 & 0,3 & 0,2 & 0,2 & 0,2 & 0,2 & 0,1 & 0,1 & 0,1 & 0,1 & 0,1 \\
\hline Total & 2,8 & 2,7 & 1,1 & 1,1 & 1,4 & 1,2 & 0,8 & 0,6 & 0,4 & 0,3 & 0,3 \\
\hline $\mathrm{VHC}^{b}$ & 1,8 & 1,9 & 1,5 & 1,6 & 1,2 & 1,6 & 0,9 & 1,0 & 0,9 & 0,7 & 0,7 \\
\hline$H_{T L V}^{b}$ & 2,1 & 1,6 & 0,8 & 0,9 & 1,1 & 0,7 & 0,5 & 0,2 & 0,2 & 0,3 & 0,1 \\
\hline Anti-HBc ${ }^{c}$ & - & - & - & 8,2 & 6,4 & 5,6 & 4,6 & 3,3 & 3,2 & 2,8 & 3,3 \\
\hline \multicolumn{12}{|l|}{ Sífilis $^{d}$} \\
\hline EIA & 2,7 & 2,2 & 2,0 & 2,0 & 1,7 & 1,5 & 1,3 & 1,9 & 1,1 & 0,9 & 0,84 \\
\hline VDRL & 0,3 & 0,2 & 0,2 & 0,3 & 0,1 & 0,1 & 0,1 & 0,1 & 0,1 & 0,1 & 0,06 \\
\hline$E I A+V D R L$ & 1,8 & 1,4 & 1,2 & 0,8 & 0,5 & 0,5 & 0,6 & 0,4 & 0,3 & 0,2 & 0,27 \\
\hline Total & 4,8 & 3,8 & 3,4 & 3,1 & 2,3 & 2,1 & 2,0 & 2,4 & 1,5 & 1,2 & 1,17 \\
\hline EIA & 0,5 & 0,6 & 0,2 & 0,1 & 0,1 & 0,07 & 0,2 & 0,8 & 0,8 & 0,3 & 0,15 \\
\hline IFI & 2,4 & 1,0 & 0,6 & 0,7 & 0,7 & 0,8 & 0,6 & 1,0 & 0,4 & 0,3 & 0,73 \\
\hline Total & 4,2 & 2,6 & 1,6 & 1,7 & 1,4 & 1,5 & 1,5 & 2,4 & 1,7 & 1,0 & 1,14 \\
\hline $\mathrm{ALT}^{f}$ & 3,8 & 3,8 & 3,5 & 4,5 & 4,1 & 3,8 & 3,5 & 3,2 & 2,6 & 2,2 & 2,3 \\
\hline Total de descarte & 20,6 & 17,6 & 13 & 22 & 19,2 & 17,7 & 14,5 & 13,8 & 10,8 & 8,8 & 9,3 \\
\hline Número de doações & 166127 & 188370 & 193440 & 198722 & 213324 & 222025 & 237981 & 247434 & 238243 & 230191 & 236001 \\
\hline
\end{tabular}

a Taxas expressas em porcentagem.

b Início em julho de 1991.

c Início em março de 1994.

d EIA = teste imunoenzimático (enzyme immunoassay); VDRL = Venereal Disease Research Laboratory slide test.

${ }^{\mathrm{e}} \mathrm{IFI}=$ imunofluorescência indireta; $\mathrm{HAl}=$ hemaglutinação indireta.

${ }^{\dagger} \mathrm{ALT}=$ aminotransferase alanina.

das a partir de março de 1994, quando da introdução do teste anti-HBc na triagem sorológica. Naquele ano, a taxa de descarte de bolsas positivas para o anti-HBc atingiu o valor de $8,2 \%$, o qual está próximo da prevalência de $\mathrm{HBc}$ verificada na população geral da Cidade de São Paulo $(12,13)$.

$\mathrm{O}$ descarte sorológico diminuiu significativamente para os testes de VIH e HTLV. Essa mudança pode ser associada à substituição dos testes baseados em lisado viral por testes recombinantes em nossa rotina. Parte dessa queda também pode ser explicada por uma possível diminuição na prevalência dessas doenças entre nossos doadores. Entretanto, ainda foi elevado o índice de descarte sorológico diretamente relacionado aos testes de anti$\mathrm{HBc}$, aminotransferase alanina (ALT),
IFI para detecção da doença de Chagas e teste ELISA para determinação de anticorpos anti-treponêmicos.

A tabela 2 mostra a porcentagem de descarte sorológico, o número de amostras confirmadas e a prevalência estimada para cada doença infecciosa nas 9942 amostras triadas entre $1^{\circ} \mathrm{e}$ 15 de novembro de 2001. O teste de HTLV foi o que apresentou o maior valor preditivo positivo, pois seis das oito amostras reativas foram confirmadas por Western blot como HTLV do tipo I. O VIH teve a menor taxa de confirmação $(12 \%)$. Isso se deve ao fato de terem sido usados dois testes de triagem na rotina. $\mathrm{O}$ mesmo aconteceu com a doença de Chagas, onde o teste de IFI foi responsável pela maioria do descarte, sendo que apenas 14 das 83 bolsas descartadas foram positivas nos três testes, enquanto três amostras foram reativas para apenas dois testes (duas para EIA e HAI e uma para EIA e IFI). Metade das amostras descartadas para VHB (48\%) apresentava o marcador anti-HBc isolado.

\section{DISCUSSÃO}

A taxa de descarte sorológico não representa a prevalência de uma determinada infecção na população de doadores de sangue; contudo, reflete um conjunto de variáveis que têm extrema importância para a qualidade do sangue. Durante o período de 11 anos analisado no presente trabalho, pudemos verificar que vários fatores contribuíram para a diminuição da taxa de descarte de bolsas na Fundação Pró- 
TABELA 2. Prevalência estimada de marcadores sorológicos em 9942 doadores de sangue da Fundação Pró-Sangue/ Hemocentro de São Paulo, Brasil, 2001

\begin{tabular}{|c|c|c|c|}
\hline Marcadores sorológicos & $\begin{array}{c}\text { Amostras } \\
\text { descartadas (\%) }\end{array}$ & $\begin{array}{l}\text { Amostras reativas nos testes } \\
\text { confirmatórios }(\%)^{\mathrm{b}}\end{array}$ & $\begin{array}{l}\text { \% Prevalência } \\
\text { (IC95\%) }\end{array}$ \\
\hline VIH (Western blot positivo) & $34(0,34)$ & $4(12)$ & $0,04(0,01-0,10)$ \\
\hline VHC (immunoblot positivo) & $68(0,68)$ & $21(31)$ & $0,21 \quad(0,13-0,32)$ \\
\hline HTLV (Western blot positivo) & $8(0,08)$ & $6(75)$ & $0,06(0,02-0,13)$ \\
\hline VHB & $349(3,51)$ & & \\
\hline Anti-HBc + HBsAg & $-c$ & $14(4)$ & $0,14(0,07-0,23)$ \\
\hline Anti-HBC + anti-HBs & $-c$ & $168(48)$ & $1,68(1,43-1,95)$ \\
\hline Anti-HBc isolado & $-c$ & $167(48)$ & $1,67(1,43-1,95)$ \\
\hline Sífilis ${ }^{d}$ & $130(1,31)$ & & \\
\hline $\mathrm{EIA}+\mathrm{FTA}-\mathrm{Abs}+\mathrm{VDRL}$ negativo & $-c^{c}$ & $81(62)$ & $0,81(0,65-1,01)$ \\
\hline EIA + FTA-Abs + VDRL positivo & $-c$ & 29 (22) & $0,29(0,20-0,42)$ \\
\hline Doença de Chagas & $83(0,83)$ & & \\
\hline Positivo em três testes ${ }^{\mathrm{e}}$ & $-c$ & $14(17)$ & $0,14(0,07-0,23)$ \\
\hline Positivo em dois testes ${ }^{\dagger}$ & $-{ }^{c}$ & $3(4)$ & $0,03(0,01-0,08)$ \\
\hline \multicolumn{4}{|c|}{$\begin{array}{l}\text { a Porcentagem do total da amostra. } \\
\text { b Porcentagem das amostras descartadas. } \\
\text { c Amostras descartadas incluídas no valor total de descarte para a doença. Anti-HBs e FTA-Abs não foram utilizados para fins de triagem. } \\
\text { d EIA = teste imunoenzimático (enzyme immunoassay); FTA-Abs = imunofluorescência indireta; VDRL = Venereal Disease Research Laboratory slide test. } \\
\text { e Teste imunoenzimático EIA, imunofluorescência indireta e hemaglutinação indireta. } \\
\text { f Teste imunoenzimático EIA + imunofluorescência indireta; ou teste imunoenzimático EIA + hemaglutinação indireta; ou imunofluorescência indireta + hemaglutinação } \\
\text { indireta. }\end{array}$} \\
\hline
\end{tabular}

Sangue/Hemocentro de São Paulo. Dentre eles, podemos ressaltar a introdução de um sistema informatizado em rede em 1994, que impediu novas doações por indivíduos com sorologia anteriormente reativa a um ou mais dos agentes etiológicos; a automação dos testes laboratoriais, iniciada em 1991 com a utilização de pipetadores automáticos, chegando à automação completa em 1999; a introdução de padrões de qualidade para certificação ISO 9000 em 1998; e as campanhas educativas que aumentaram, sobremaneira, a porcentagem de doadores de repetição. Em 1991, os doadores de repetição eram $10 \%$ do total de doadores. Em 2001, passaram a representar $50 \%$ do total de doadores. Apesar dos vários fatores que contribuíram para tal melhoria, verificamos que a taxa de descarte de bolsas ainda é significativamente maior do que aquela observada em países desenvolvidos, como por exemplo os Estados Unidos, onde o descarte sorológico está em torno de $4 \%$ (6). Atualmente, $50 \%$ dos indivíduos que procuram os nossos serviços estão fazendo sua primeira doação, enquanto que, nos Estados Unidos, ape- nas 19\% dos doadores apresentam este perfil (5).

Pudemos observar que os nossos dados acerca de prevalência de doenças infecciosas estão abaixo da prevalência registrada para a população geral $(13,14)$. Essa diferença pode ser decorrente da triagem prévia do doador de sangue, que pode ser excluído quando admite qualquer comportamento de risco para uma ou mais doenças infecciosas. Outro fato que contribui para reforçar tal diferença é, justamente, o perfil do doador que atualmente procura nosso banco de sangue, prevalecendo os doadores de repetição em detrimento daqueles que vão doar sangue pela primeira vez. A diferença entre os doadores da Fundação Pró-Sangue e outros grupos da população pode ser exemplificada pelo VIH, cuja prevalência foi de $0,04 \%$ entre nossos doadores contra $0,8 \%$ entre gestantes (14).

Outros estudos enfatizam essa diferença. Foccacia et al. (13) determinaram prevalências de 1,4 e $5,9 \%$ para anti-VHC e anti-VHB, respectivamente, na população geral da Cidade de São Paulo. Sabino et al. (15), em estudos de gestantes, puderam verificar uma prevalência de $9 \%$ de anti-HBc nessa população específica. Nossos dados, no entanto, revelaram valores de prevalência inferiores para o anti-VHC $(0,21 \%)$ e para o anti-HBc $(1,67 \%)$ entre os doadores de sangue. Os dados de prevalência na Fundação Pró-Sangue também foram menores do que os descritos por Treitinger et al. (10) para doadores de sangue em uma cidade do sul do país, Florianópolis (Estado de Santa Catarina) - 0,7 e 9,3\%, respectivamente, para anti-VHC e anti-HBc. A prevalência de $\mathrm{VHB}$ varia entre os estados do Brasil; além disso, é possível que a porcentagem de pessoas que doan sangue pela primeira vez seja maior naquele banco.

Ao comparar os dados de prevalência da Fundação Pró-Sangue em anos anteriores com os obtidos neste estudo, pode-se observar uma diminuição da prevalência de algumas doenças infecciosas. Assim, analisando a prevalência do HTLV entre os doadores de sangue, encontramos $0,17 \%$ de positividade em 1996 (15) contra 0,06\% no presente estudo. $\mathrm{O}$ mesmo perfil foi observado para a doença de Chagas. 
Considerando a reatividade a três testes para confirmação de infecção, verifica-se que esse índice caiu de 0,7\% em 1991 para 0,14\% em 2001.

Existem, ainda, poucos estudos sobre a porcentagem de descarte e prevalência de infecção em outros países da América Latina. Recentemente, Schmunis et al. (16) sistematizaram os dados de descarte sorológico de 12 países da América Latina para VHC, VIH, VHB e a doença de Chagas no período de 1994 a 1997. Com exceção da doença de Chagas, cuja prevalência varia consideravelmente entre os países, os dados apresentados naquele estudo são semelhantes aos encontrados no Brasil.

Em conclusão, nossos dados mostram a diminuição dos índices de descarte de bolsas de sangue a partir dos testes de triagem sorológica. Também ficou evidente a diminuição da pre- valência de doenças infecciosas entre os indivíduos que procuram, atualmente, o nosso serviço de doação de sangue. Tais fatos refletem o sucesso de um trabalho amplo, que abrange diversas áreas do ciclo do sangue, cujo resultado final é a conquista de um número significativamente mais elevado de doadores de repetição e, por conseguinte, o aumento da qualidade do sangue.

\section{REFERÊNCIAS}

1. Gretch DR. Diagnostic tests for hepatitis C. Hepatology 1997;26(3 Suppl 1):43S-47S.

2. Zaaijer HL, Exel-Oehlers P, Kraaijeveld T, Altena E, Lelie PN. Early detection of antibodies to HIV-1 by third-generation assays. Lancet 1992;340(8822):770-772.

3. Williams LO, Blumer SO, Schalla WO, Robinson $\mathrm{PH}$, Handsfield JH, Fehd RJ, et al. Laboratory performance in HTLV-I/II analysis. Transfusion 2000;40(12):1514-1521.

4. ANVISA. Serviços de hemoterapia: relatórios de produção. Disponível em http://www.anvisa. gov.br/sangue/hemoterapia/producao.htm. Acessado em 2002.

5. Glynn SA, Kleinman SH, Schreiber GB, Busch MP, Wright DJ, Smith JW, et al. Trends in incidence and prevalence of major transfusiontransmissible viral infections in US blood donors, 1991 to 1996. Retrovirus Epidemiology Donor Study (REDS). JAMA 2000;284(2):229235 .

6. Ownby HE, Korelitz JJ, Busch MP, Williams $\mathrm{AE}$, Kleinman $\mathrm{SH}$, Gilcher RO, et al. Loss of volunteer blood donors because of unconfirmed enzyme immunoassay screening results. Retrovirus Epidemiology Donor Study. Transfusion 1997;37(2):199-205.

7. Galvão-Castro B, Loures L, Rodrigues LG, Sereno A, Ferreira Junior OC, Franco LG, et al. Distribution of human T-lymphotropic virus type I among blood donors: a nationwide Brazilian study. Transfusion 1997;37(2):242-243.

8. Monteiro de Castro MS, Assunção RM, Proietti FA. Spatial distribution of the human Tlymphotropic virus types I and II (HTLV-I/II) infection among blood donors of Hemominas Foundation, Belo Horizonte, Minas Gerais State, Brazil, 1994-1996. Cad Saude Publica 2001;17(5):1219-1230.

9. Kupek EJ. HIV seroprevalence among blood donors in southern Brazil in the decade of 1990. Braz J Infect Dis 2000;4(5):217-225.

10. Treitinger A, Spada C, Ferreira LA, Neto MS, Reis M, Verdi JC, et al. Hepatitis B and hepatitis $\mathrm{C}$ prevalence among blood donors and HIV-1 infected patients in Florianópolis - Brazil. Braz J Infect Dis 2000;4(4):192-196.

11. Zicker F, Martelli CM, Andrade AL, Almeida e Silva S. Trends of T. cruzi infection based on data from blood bank screening. Rev Inst Med Trop Sao Paulo 1990;32(2):132-137.

12. Sabino EC, Guerra EM, Oba IT, Spina AM, Vaz AJ. The incidence of hepatitis B markers in pregnant women at their first consultation in metropolitan-area health centers, São Paulo, Brazil. Rev Inst Med Trop Sao Paulo 1992;34(6):535-541.

13. Focaccia R, da Conceição OJ, Sette $\mathrm{H} \mathrm{Jr}$, Sabino E, Bassit L, Nitrini DR, et al. Estimated prevalence of viral hepatitis in the general population of the municipality of São Paulo, measured by a serologic survey of a stratified, randomized and residence-based population. Braz J Infect Dis 1998;2(6):269-284.

14. Miranda AE, Alves MC, Neto RL, Areal KR Gerbase AC. Seroprevalence of HIV, hepatitis $B$ virus, and syphilis in women at their first visit to public antenatal clinics in Vitoria, Brazil. Sex Transm Dis 2001;28(12):710-713.

15. Sabino EC, Zrein M, Taborda CP, Otani MM Ribeiro dos Santos G, Sáez-Alquézar A. Evaluation of the INNO-LIA HTLV I/II assay for confirmation of human T- cell leukemia virusreactive sera in blood bank donations. J Clin Microbiol 1999;37(5):1324-1328.

16. Schmunis GA, Zicker F, Cruz JR, Cuchi P. Safety of blood supply for infectious diseases in Latin American countries, 1994-1997. Am J Trop Med Hyg 2001;65(6):924-930.

Manuscrito recebido em 11 de fevereiro de 2002. Aceito em versão revisada em 5 de agosto de 2002 
ABSTRACT Objectives. To analyze the changes in the proportion of blood units discarded from 1991 through 2001 at the Pro-Blood Foundation/Blood Center of São Paulo (Fundação Pró-Sangue/Hemocentro de São Paulo), which is the largest blood bank in Latin America,

The discarding of blood units and the prevalence of infectious diseases in donors at the Pro-Blood Foundation/Blood Center of São Paulo, São Paulo, Brazil and to determine the prevalence of infectious diseases among donors at the Blood Center in November 2001.

Methods. We compiled data concerning the discarding of blood units due to the presence of serological markers for communicable diseases at the Blood Center during the period from 1991 through 2001. To determine the prevalence of infectious diseases, 9942 screened samples were analyzed in November 2001; all reactive samples underwent confirmatory tests.

Results. Over the study period there was a significant decrease in the percentage of units discarded, from $20 \%$ in 1991 to $9 \%$ in 2001. In November 2001 the prevalence of infectious diseases among donors was: $0.04 \%$ for human immunodeficiency virus (HIV), $0.21 \%$ for hepatitis C virus (HCV), $0.06 \%$ for human T-lymphotropic virus (HTLV), $0.14 \%$ for Chagas' disease, and $1.10 \%$ for syphilis. For hepatitis B virus, the prevalences found were: $0.14 \%$ for anti-HBc and HBsAg, $1.68 \%$ for anti-HBc and antiHBs, and $1.67 \%$ for isolated anti-HBc.

Conclusions. The decrease in the discarding of blood units and in infectious diseases among donors at the Blood Center of São Paulo reflects the increase in the Center's percentage of repeat donors.

\title{
Caribbean Health Research Council 48th Annual Scientific Meeting
}

\author{
Dates: $\quad$ 1-3 May 2003 \\ Location: Wyndham Nassau Resort \& Crystal Palace Casino \\ Nassau, Bahamas
}

The 48th Annual Scientific Meeting of the Caribbean Health Research Council (CHRC) will serve as a forum at which dozens of Caribbean researchers will present their findings. The event will include both oral and poster presentations as well as satellite sessions. There will also be three special lectures on Caribbean health concerns, with distinguished speakers talking on HIV/AIDS in the Caribbean, why infections are emerging in the new millennium, and the evidence on which therapies should be based.

There will be 80 oral and 69 poster presentations, covering such topics as HIV/AIDS, nutrition, communicable diseases, child health, surgical studies, family health, chronic noncommunicable diseases, health services research, mental health, and medical education.

There will also be two satellite meetings on the afternoon of Friday, 2 May. A meeting of the National Institute on Drug Abuse (NIDA) and the Caribbean Epidemiology Centre (CAREC) will have as its theme "Twin Epidemics of Substance Use and HIV/AIDS in the Caribbean: Research as a Resource to a Major Public Health Problem." The other satellite gathering will be a meeting of the Caribbean Association of Laboratory Medicine.

The CHRC Annual Meeting is intended for researchers, health planners, physicians, and other health personnel. The official language of the event is English. Conference registration is free for medical students, and for all others the registration fee is US\$ 150 if paid by March 272003 and US\$ 180 after that date.

\author{
Information: \\ Dr. Donald T. Simeon, Director of Research \\ Caribbean Health Research Council \\ 25A Warner Street, St. Augustine, Trinidad and Tobago \\ Tel: (868) 645-3769 or 645-0705 • Fax: (868) 645-0705 \\ E-mail: chrc@trinidad.net \\ Web site: http://www.carec.org
}

